\title{
THE EXISTENCE OF COMPLETE RIEMANNIAN METRICS
}

\section{KATSUMI NOMIZU ${ }^{1}$ AND HIDEKI OZEKI}

The purpose of the present note is to prove the following results. Let $M$ be a connected differentiable manifold which satisfies the second axiom of countability. Then (i) $M$ admits a complete Riemannian metric; (ii) If every Riemannian metric on $M$ is complete, $M$ must be compact.

In fact, somewhat stronger results will be given as Theorems 1 and 2 below.

Let $M$ be a connected differentiable manifold. It is known that if $M$ satisfies the second axiom of countability, then $M$ admits a Riemannian metric. Conversely, it can be shown that the existence of a Riemannian metric on $M$ implies that $M$ satisfies the countability axiom. For any Riemannian metric $g$ on $M$, we can define a natural metric $d$ on $M$ by setting the distance $d(x, y)$ between two points $x$ and $y$ to be the infinimum of the lengths of all piecewise differentiable curves joining $x$ and $y$. The Riemannian metric $g$ is complete if the metric space $M$ with $d$ is complete. It is known that this is the case if and only if every bounded subset of $M$ (with respect to $d$ ) is relatively compact.

We shall say that a Riemannian metric $g$ is bounded if $M$ is bounded with respect to the metric $d$. We shall prove

Theorem 1. For any Riemannian metric $g$ on $M$, there exists a complete Riemannian metric which is conformal to $\mathrm{g}$.

TheOREM 2. For any Riemannian metric $g$ on $M$, there exists a bounded Riemannian metric which is conformal to $\mathrm{g}$.

The result (ii) mentioned in the beginning is a consequence of Theorem 2, because if a bounded Riemannian metric, which exists on $M$, is complete, then $M$ itself is compact.

Proof of Theorem 1. At each point $x$ of $M$, we define $r(x)$ to be the supremum of positive numbers $r$ such that the neighborhood $S(x, r)=\{y ; d(x, y)<r\}$ is relatively compact. If $r(x)=\infty$ at some point $x, M$ is compact and hence $g$ is complete. Assume therefore that $r(x)<\infty$ for every $x$. It is easy to verify that $|r(x)-r(y)| \leqq d(x, y)$ for all $x$ and $y$ in $M$, which shows that $r(x)$ is a continuous function on

Received by the editors November 14, 1960.

1 Partially supported by NSF grant 14032. 
$M$. Since $M$ satisfies the second axiom of countability, ${ }^{2}$ we can choose a differentiable function $\omega(x)$ such that $\omega(x)>1 / r(x)$ at every point $x$. We define a conformal Riemannian metric $g^{\prime}$ by $g_{x}^{\prime}=(\omega(x))^{2} g_{x}$ at every point $x$.

In order to prove that $g^{\prime}$ is complete, we shall show that $S^{\prime}(x, 1 / 3)$ $=\left\{y ; d^{\prime}(x, y)<1 / 3\right\}$ is contained in $S(x, r(x) / 2)$ (and hence relatively compact) for every $x$, where $d^{\prime}$ is the distance defined by $g^{\prime}$. For this purpose, assume $d(x, y) \geqq r(x) / 2$. For any piecewise differentiable curve $x(t), a \leqq t \leqq b$, joining $x$ and $y$, its length $L=\int_{a}^{b}\|d x / d t\| d t(\|d x / d t\|$ denotes the length of the tangent vector $d x / d t$ with respect to $g$ ) is not smaller than $d(x, y)$ and hence $L \geqq r(x) / 2$. We evaluate the length $L^{\prime}$ of the same curve with respect to $g^{\prime}$. By a mean value theorem, we have

$$
\begin{gathered}
L^{\prime}=\int_{a}^{b} \omega(x)\|d x / d t\| d t \omega(x(c)) L \\
>L / r(x(c)),
\end{gathered}
$$

where $c$ is a number between $a$ and $b$. Since $|r(x(c))-r(x)|<d(x, x(c))$ $\leqq L$, we have $r(x(c))<r(x)+L$ so that $L^{\prime}>L /(r(x)+L)$. Since $L \geqq r(x) / 2$, we have $L^{\prime}>1 / 3$. Therefore $d^{\prime}(x, y) \geqq 1 / 3$. This proves that $S^{\prime}(x, 1 / 3)$ is contained in $S(x, r(x) / 2)$.

Proof of Theorem 2. By virtue of Theorem 1, we may assume that the given Riemannian metric $g$ is complete. Let $o$ be an arbitrarily fixed point of $M$. The function $d(x, o)$ is continuous. Let $\omega(x)$ be a differentiable function such that $\omega(x)>d(x, o)$ on $M$. We shall prove that the Riemannian metric $g^{\prime}=e^{-2 \omega(x)} g$ is bounded. Let $x$ be an arbitrary point of $M$. Since $g$ is complete, there exists a minimizing geodesic $C$ from $o$ to $x$, that is, a geodesic $C$ whose length $L$ is equal to $d(x, o)$. Let $x(s)$ be a parametric representation of $C$ in terms of the arc length measured from $o$. Since any subarc of $C$ is a minimizing geodesic between its end points, we have $d(x(s), o)=s$ for every $s$. The length of the tangent vector $d x / d s$ with respect to $g^{\prime}$ is equal to

\footnotetext{
${ }^{2}$ This fact, mentioned in the introduction, can be proved, for example, as follows. When $M$ is not compact, we define for every natural number $n$ a neighborhood $U_{n}(x)=\{y ; d(x, y)<r(x) / n\}$ for each point $x$. It is easy to verify that $\left\{U_{n}\right\}$ defines a uniform structure on the space $M$ and that $M$ is uniformly locally compact (i.e., there is some $n$, indeed $n=2$ will do in this case, such that $U_{n}(x)$ is relatively compact for every $x$ ). Since $M$ is connected, it follows that $M$ is the sum of countably many compact subsets. Now for a differentiable manifold, this means that it satisfies the second axiom of countability.
} 
$e^{-\omega(x(s))}$. The length $L^{\prime}$ of $C$ with respect to $g^{\prime}$ is thus $\int_{0}^{L} e^{-\omega(x(s))} d s$. Since $\omega(x(s))>d(x(s), 0)=s$, we have

$$
L^{\prime}<\int_{0}^{L} e^{-s} d s<\int_{0}^{\infty} e^{-s} d s=1,
$$

which implies that $d^{\prime}(x, 0)<1$ for every $x$.

BROWN UNIVERSITY AND

BRANDEIS UNIVERSITY 